\title{
Caracterização físico-química e microbiológica do esgoto da UEPB com proposta de tratamento para fins de reuso
}

\section{Physicochemical and microbiological characterization of wasterwater of the UEPB with proposed of treatment for reuse purposes}

\author{
Matheus Urtiga Sousa ${ }^{1 *}$; Lígia Maria Ribeiro Lima ${ }^{2}$
}

\begin{abstract}
Resumo: Nos últimos anos, a preocupação com as questões ambientais vem sendo bastante discutidas, onde merece destaque, o descarte indevido do esgoto doméstico no meio ambiente, portanto o presente estudo objetivou realizar a caracterização físico-química e microbiológica do esgoto descartado no solo, no Centro de Ciências e Tecnologia da Universidade Estadual da Paraíba, campus de Campina Grande - PB. Para tanto foram desenvolvidas análises físico-químicas, tais como pH, Fósforo Total, DBO, DQO, Nitratos, Nitritos, Nitrogênio Amoniacal, Orto-Fosfatos e Alcalinidade, bem como análise microbiológica (coliformes). Observou-se que o efluente na unidade em questão não recebe tratamento para seu descarte, sendo identificadas características físico-químicas e microbiológicas de esgoto in natura. Faz-se necessário desta forma, investimentos em análises periódicas para assim realizar um tratamento adequado ao efluente visando-se reduzir os riscos ambientais e de saúde pública. Após os resultados das análises, foi proposto, além do tratamento preliminar, um tratamento biológico para o efluente, do tipo, lagoa facultativa primária seguida de duas lagoas de maturação, onde a eficiência do tratamento nos parâmetros analisados se enquadra aos padrões estabelecidos pelo CONAMA 430/2011, na qual o efluente pós - tratado poderia ser reutilizado para fins menos nobres, destinando a água potável para atividades que requerem uma qualidade melhor.
\end{abstract}

Palavras-chaves: Esgoto bruto; Coliformes; Tratamento biológico.

Abstract: In recent years, concern about environmental issues has been widely discussed, which deserves mention the improper disposal of domestic sewage into the environment, so this study aimed to perform the physicochemical and microbiological sewage discarded the soil, in the Science and Technology Center of the State University of Paraiba, campus of Campina Grande - PB. Therefore, we developed physical-chemical analysis such as pH, Total Phosphorus, BOD, COD, Nitrate, Nitrite, Ammonia Nitrogen, Orto-Phosphates and alkalinity, as well as microbiological analysis (coliforms). It was noted that the effluent in the unit in question does not receive treatment for their disposal, identified physical, chemical and microbiological characteristics of raw sewage. It is necessary therefore, investment in periodic reviews thus to carry out an appropriate treatment to effluent aiming to reduce environmental risks and public health. After the analysis results, it was proposed, in addition to preliminary treatment, a biological treatment for the effluent, type, primary facultative pond followed by two ponds maturation, in which the treatment efficiency in the analyzed parameters falls within the standards set by CONAMA 430/2011 in which the effluent post-treaty could be reused for less noble purposes, intended to clean water for activities that require a better quality.

Key words: Raw sewage; Coliforms; Biological treatment.

\footnotetext{
*Autor para correspondência

Recebido para publicação em 27/10/2015; aprovado em 20/11/2015

${ }^{1}$ Engenheiro Sanitarista e Ambiental - CCT - UEPB - E-mail: matheusurtiga@ gmail.com

${ }^{2}$ Doutora em Engenharia de Processos - CCT - UFCG - E-mail: ligiauepb@gmail.com
} 


\section{INTRODUÇÃO}

Nos últimos anos a preocupação com as questões ambientais vem sendo bastante discutida, uma vez que o desequilíbrio gerado de uma forma global encaminha-se ao surgimento de grandes impactos ambientais negativos na sociedade. Umas dessas problemáticas são resultantes do descarte indevido de esgoto doméstico no meio ambiente (PALMEIRA, 2014).

Esgotos são definidos como águas que, após consumo humano (uso doméstico, industrial ou comercial) apresentam alterações nas características naturais.

Segundo a NBR 9648 (ABNT, 1986), esgoto sanitário é o despejo líquido constituído de esgotos doméstico e industrial, água de infiltração e a contribuição pluvial parasitária.

As águas residuais são compostas de 99,9\% de água, sendo que $0,1 \%$ restantes compreendem sólidos orgânicos e inorgânicos dissolvidos e suspensos, bem como microrganismos (VON SPERLING, 2002).

Os lançamentos de esgotos de origem urbana em cursos d'água são um dos principais fatores responsáveis pela degradação da qualidade do ambiente aquático, bem como pelo surgimento de efeitos tóxicos à comunidade do referido ambiente. A Legislação Brasileira não permite que o efluente lançado cause ou possua potencial para causar efeitos tóxicos aos organismos aquáticos, requerendo testes de toxicidade com espécies representativas de pelo menos dois níveis tróficos. Ademais, os esgotos produzidos pelas atividades de uma Universidade podem apresentar elevado potencial de toxicidade, devido à complexidade da composição destes efluentes (AQUINO, 2013).

É válido ressaltar a importância de se tratar as águas residuais, haja vista a proliferação de vetores parasitários e infecciosos, bem como, a degradação dos corpos de água, tornando-as com qualidade bastante inferior ao natural. As substâncias presentes no esgoto desempenham função deletéria aos corpos de água, a saber: Diminuição do oxigênio dissolvido, provocando a morte de inúmeras espécies aquáticas; emanação de odores, bem como, aumento da turbidez da água.

O sistema de tratamento associado à reciclagem de esgotos, em descargas sanitárias e lavagens de pisos, poderia suprir a escassez de água necessária para realizar tais atividades. Parcerias poderiam potencializar e sensibilizar sobre o uso racional da água, bem como, o reuso de esgotos tratados para diversos fins, contribuindo assim para o meio ambiente, uma vez que são práticas corretas, o que já é adotado em alguns países, mais especificamente, os do Oriente Médio (RIBEIRO, 2014)

O reuso de águas residuais ou residuárias tratadas não é um conceito meramente do cotidiano, estudos relatam que esse tipo de prática surgiu na Grécia antiga a milhares de anos, diante de um cenário de escassez hídrica. Nesse sentido, é possível considerar o reuso da água como parte integrante de um conceito mais abrangente, que é o uso racional desse recurso, na qual compreende o controle de perdas e desperdícios, bem como, a redução do consumo demasiado (AMBIENTE BRASIL, 2014).

Existem várias formas de reuso de água, onde se pode destacar: Irrigação de campos de cultivos, recarga de aquíferos, aumento da vazão de cursos de água e aquicultura (HESPANHOL, 2002).

Portanto o objetivo desse trabalho foi caracterizar o esgoto descartado no Centro de Ciências e Tecnologia (CCT) da UEPB, propondo um sistema de tratamento para este para fins de reuso na própria instituição.

\section{MATERIAL E MÉTODOS}

O sistema experimental para recepção do esgoto descartado foi constituído por um reservatório de PVC com volume de 1000 litros (Figura 1), onde estava sendo lançado esgoto advindo do Centro de Ciências e Tecnologia (CCT), da Universidade Estadual da Paraíba (UEPB), localizado no Bloco C.

Figura 1. Sistema experimental para recepção do esgoto descartado

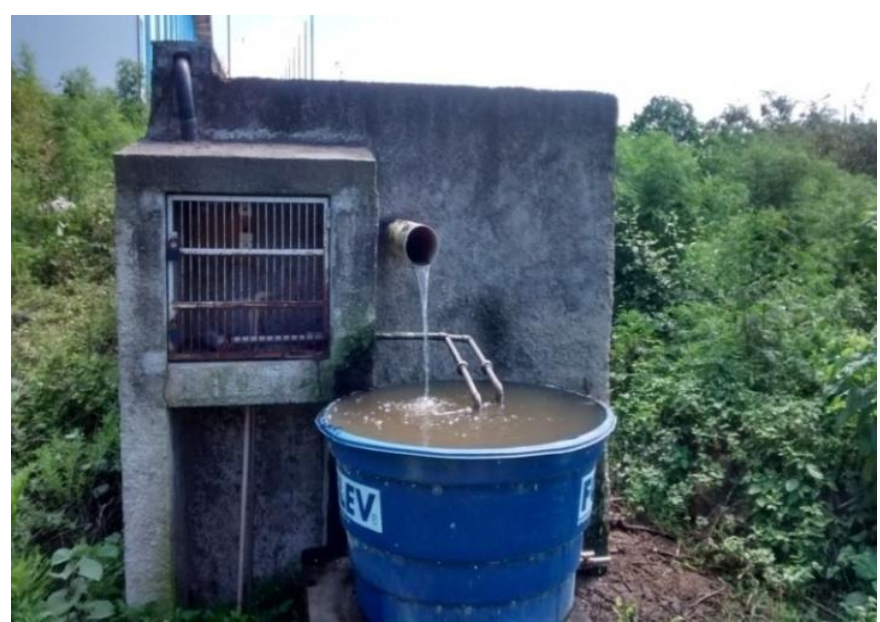

Fonte: Autor (2014)

As etapas de coletas e análises foram executadas nos meses de setembro e outubro de 2014, na qual, foram feitas leituras acerca das características físicas, químicas e microbiológicas do esgoto descartado no Bloco C/CCT/UEPB.

As coletas foram iniciadas no dia 04 de setembro de 2014 pela manhã, utilizando-se recipiente de vidro de cor âmbar de 1 litro, previamente esterilizado, para que não ocorressem interferências externas nos futuros resultados. Em seguida, levou-se a amostra de esgoto coletada, para o laboratório de Saneamento Ambiental, localizado no mesmo bloco, para assim realizar a análise do $\mathrm{pH}$ do esgoto descartado.

As demais análises físico-químicas foram desenvolvidas na Estação Experimental de Tratamento Biológico de Esgoto Sanitário (EXTRABES), tais como: Nitrito, Nitrato, DQO, Alcalinidade, Nitrogênio Amoniacal, Fósforo Total, OrtoFosfato. No período de setembro a outubro de 2014, foram realizadas três análises de cada parâmetro (triplicata). A amostra era coletada no período da manhã e à tarde, para o procedimento das análises que eram realizadas no mesmo dia, ou seja, toda quinta-feira, porém os outros dias da semana eram destinados para preparo dos materiais e reagentes.

A análise de DBO foi feita no laboratório de Controle de Qualidade do CTCC/SENAI. Foi coletada a amostra pela manhã em recipiente âmbar (recipientes de 1 litro cada), para a análise que teve a duração de cinco dias. 
A análise microbiológica foi realizada parte no laboratório de Saneamento Ambiental da UEPB, e parte na EXTRABES. O recipiente da amostra era previamente esterilizado, juntos com os tubos de ensaio para o procedimento da análise, a fim de eliminar os microrganismos ali que possam a vir existir. A coleta da amostra era sempre feita pela manhã e a análise realizada logo em seguida, para que os meios externos não pudessem a vir causar interferências no resultado final.

As análises foram realizadas obedecendo aos cuidados e técnicas apropriadas de acordo com o Manual de Análises Físico-químicas de Águas de Abastecimento e Residuárias (2001), bem como no Manual de Procedimentos e Técnicas Laboratoriais voltados para analises de água, esgoto sanitário e industrial (2004).

\section{RESULTADOS E DISCUSSÃO}

\section{Análises Físico-Químicas:}

$$
\text { - pH }
$$

$\mathrm{Na}$ análise de $\mathrm{pH}$, a média foi de 7,22. No caso de emprego de lagoas de estabilização certamente irá ocorrer um aumento de $\mathrm{pH}$, haja vista, que o mesmo está atrelado à atividade das algas, na qual consomem o dióxido de carbono $\left(\mathrm{CO}_{2}\right)$ presente no efluente, utilizando para seu metabolismo vital, e libera radicais hidroxilas $\left(\mathrm{OH}^{-}\right)$, com isso elevando o $\mathrm{pH}$. A elevação do $\mathrm{pH}$ também está atrelada às condições ambientais, ação dos ventos, sobrecarga orgânica e operacionalização das lagoas.

Segundo Palmeira (2014) o aumento da média de $\mathrm{pH}$ entre o esgoto bruto ( $\mathrm{pH} 7,22)$ e a lagoa de estabilização $(\mathrm{pH}$ 8,08) é de 11,91\% (Lagoa Facultativa-Lagoa de MaturaçãoLagoa de Maturação). De acordo com Von Sperling (2002), o pH médio das lagoas de estabilização é de 8 , apresentando caráter alcalino, o que se encontra em consonância aos padrões estabelecidos pela resolução vigente, a saber: CONAMA 430/2011, onde relata que para lançamentos de efluentes em corpos de água, deve estar entre 5 e 9.

\section{- Nitrogênio Amoniacal}

A concentração média de nitrogênio amoniacal do efluente foi de $33,206 \mathrm{mg} . \mathrm{L}^{-1}$, estando dentro da faixa prevista para esgoto bruto (12-50 mg. $\mathrm{L}^{-1}$ ), de acordo com Metcalf e Eddy (1991). Segundo Júnior et al. (2000), caso seja utilizado um tratamento por lagoas de estabilização do tipo de uma lagoa facultativa seguida de duas de maturação, haverá uma tendência de decaimento desta concentração, na ordem de $80 \%$, em escala piloto. Já Silva et al. (2010), em um mesmo sistema (Lagoa Facultativa - Lagoa de Maturação - Lagoa de Maturação), apresentou remoção de nitrogênio amoniacal da ordem de 57\%. Palmeira (2014) apresentou uma remoção de $55 \%$ de nitrogênio amoniacal em um mesmo sistema.

A remoção de nitrogênio amoniacal no sistema de lagoas de estabilização está diretamente atrelada ao processo de assimilação do nitrogênio amoniacal pela biomassa das algas, bem como, ao processo de nitrificação e desnitrificação (pouco representativo) e ao processo de volatilização $(\mathrm{pH}>8)$, sendo esse último considerado por muitos autores, como principal mecanismo de remoção de nitrogênio em lagoas de estabilização (JÚNIOR et al., 2000), o que explica diferentes eficiências em um mesmo sistema.

Pode-se concluir que quanto ao lançamento em corpos hídricos, o efluente em questão, quando for submetido ao tratamento biológico por lagoas de estabilização, baseando-se nas eficiências de remoção, apresentará uma concentração final abaixo de 20,0 mg. $\mathrm{L}^{-1}$, o que atende aos parâmetros exigidos pela resolução (CONAMA 430/11).

\section{- Nitrato}

A concentração média de nitrato foi de $0,379 \mathrm{mg} \cdot \mathrm{L}^{-1}$, o que encontra-se dentro da faixa para esgoto doméstico que é de 0,10 - 0,40 mg/L, segundo (Jordão e Pessoa, 2005), o que a torna desprezível, quando comparada a outras formas de nitrogênio presentes no esgoto bruto. Caso seja utilizado um tratamento por lagoas de estabilização do tipo: Lagoa Facultativa - Lagoa de Maturação - Lagoa de Maturação, em escala piloto; haverá uma tendência de decaimento desta concentração, devido à nitrificação em lagoas de estabilização, porém em escala desprezível (JÚNIOR et al., 2000).

Atualmente não existe parâmetro mínimo de concentração de nitrito para lançamento em corpos hídricos, segundo a Resolução CONAMA 430 (2011).

\section{- Nitrito}

A concentração média de nitrito foi de $0,023 \mathrm{mg} \cdot \mathrm{L}^{-1}$, o que encontra-se dentro da faixa para esgoto doméstico que é de 0,0 - 0,10 mg/L, segundo (Jordão e Pessoa, 2005), o que a torna desprezível, quando comparada a outras formas de nitrogênio presentes no esgoto bruto. No caso seja utilizado um tratamento por lagoas de estabilização (LF - LM - LM), em escala piloto, haverá uma tendência de decaimento desta concentração, devido à nitrificação em lagoas de estabilização, porém em escala desprezível (JÚNIOR et al., 2000).

Atualmente não existe parâmetro mínimo de concentração de nitrito, para lançamento em corpos hídricos, segundo a Resolução CONAMA 430 (2011). Porém sabe-se que em concentrações altas (bem acima de $1 \mathrm{mg} . \mathrm{L}^{-1}$ ) é bastante tóxico. (AYRES; WESTCOT, 1985).

\section{- Alcalinidade}

A concentração média da alcalinidade do esgoto do CCT da UEPB foi de $342,45 \mathrm{mg}$ de $\mathrm{CaCO}_{3} \cdot \mathrm{L}^{-1}$. A alcalinidade tende a diminuir no decorrer das lagoas de estabilização, tal fato pode ser explicado pela presença das algas nas lagoas, na qual assimilam os íons bicarbonatos $\left(\mathrm{HCO}_{3}{ }^{-}\right)$, convertendo-os em dióxido de carbono $\left(\mathrm{CO}_{2}\right)$, utilizando para seu metabolismo vital e liberando raciais hidroxilas $\left(\mathrm{OH}^{-}\right)$para o meio líquido. As lagoas de estabilização, do tipo Lagoa Facultativa - Lagoa de Maturação - Lagoa de Maturação, apresentam uma eficiência média de redução da alcalinidade na ordem de $0,532 \%$, baseado nos dados de PALMEIRA (2014).

Segunda a resolução CONAMA 430 (2011), não existe uma concentração mínima de alcalinidade para parâmetros de lançamentos de efluentes em corpos hídricos.

\section{- Fósforo Total}

A presença de fósforo sob forma de fosfatos nos esgotos está diretamente ligada a produtos de limpeza, principalmente detergentes, e em menor concentração à carnes e alimentos.

A concentração média de fósforo total no efluente foi de 7,26 mg. $\mathrm{L}^{-1}$, o que está dentro da faixa estabelecida para esgoto bruto $\left(6,5-9,0 \mathrm{mg} . \mathrm{L}^{-1}\right)$, segundo Araújo (1993). No caso de emprego em lagoas de estabilização em escala reduzida, do tipo LF - LM - LM (proposto), a concentração 
de fósforo total tende a diminuir, podendo ser constatado uma eficiência média de remoção da ordem de praticamente $0,0 \%$ (Júnior et al., 2000). Silva et al. (2010) constataram uma eficiência média de remoção da ordem de praticamente 30\%; já em estudos desenvolvidos por Palmeira (2014), a remoção de fósforo total foi de 33\%, enquanto Von Sperling (2002) destacou que a remoção não ultrapassa $35 \%$.

Tal fato pode ser explicado devido o fósforo constituir um dos principais nutrientes dos microrganismos aquáticos e plantas (SAWYER et al., 1994). A principal forma de remoção de fósforo em lagoas de estabilização é a precipitação de fosfatos em condições de elevado $\mathrm{pH}$ (JÚNIOR et al., 2000), bem como a assimilação destes pela biomassa das algas presentes no sistema, o que explicam-se diferentes eficiências na remoção do fósforo total. Segunda a resolução CONAMA 430 (2011), não existe uma concentração mínima de fósforo total para parâmetros de lançamentos de efluentes em corpos hídricos ou a reutilização do efluente tratado. Vale salientar que se o fósforo for descarregado, continuamente, sem o devido tratamento biológico, poderá ocorrer um processo, conhecido por eutrofização.

\section{- Orto-Fosfato}

A concentração média de orto-fosfato no esgoto da UEPB foi de $3,75 \mathrm{mg} . \mathrm{L}^{-1}$, o que está dentro da faixa estabelecida para esgoto bruto (2,0 a 7,0 mg. $\left.\mathrm{L}^{-1}\right)$, de acordo com Araújo (1993). Quando esse efluente for submetido a um processo de tratamento biológico por lagoas de estabilização em escala piloto, do tipo: Lagoa Facultativa - Lagoa de Maturação - Lagoa de Maturação, irá ocorrer uma remoção média da ordem de $81 \%$ (SILVA et al., 2010). Segundo os dados apresentados por Palmeira (2014), o sistema apresentou uma eficiência de 47,81\%.

As algas assimilam o orto-fosfato solúvel e o incorporam a sua biomassa, pois a forma solúvel do fósforo (orto-fostato) compõe parte do material celular das algas (SILVA et al., 2010). Para tanto, fatores como $\mathrm{pH}$, carga orgânica aplicada e tempo de detenção hidráulica, influenciam na remoção de orto-fosfato, o que explicam-se as diferentes eficiências para um mesmo sistema.

\section{- Demanda Química de Oxigênio (DQO)}

A concentração média da Demanda Química de Oxigênio foi $558,57 \mathrm{mg} \mathrm{de} \mathrm{O}_{2} \cdot \mathrm{L}^{-1}$, o que está dentro da faixa para esgoto bruto $\left(400-1500 \mathrm{mg}\right.$ de $\left.\mathrm{O}_{2} \cdot \mathrm{L}^{-1}\right)$, segundo Silva e Mara (1979). No entanto, quando esse efluente for submetido a um processo de tratamento biológico por lagoas de estabilização em escala piloto, do tipo Lagoa Facultativa Lagoa de Maturação - Lagoa de Maturação, irá ocorrer uma remoção média da DQO da ordem de $35 \%$ (PALMEIRA, 2014). Segundo Sperling (2002), um sistema de lagoas de estabilização, apresenta uma remoção de $70 \%$ de DQO. Vale (2006), em um mesmo sistema (LF - LM - LM), apresentou uma remoção de 60 e 78\%, no Sistema Santa Lola 1 e Sistema Beira - Rio, respectivamente.

A DQO expressa o quantitativo de matéria orgânica passível de bioestabilização, portanto, o tempo de detenção hidráulica e carga orgânica aplicada, bem como, incidência de luz e profundidade das lagoas de estabilização, influenciarão diretamente na eficiência de remoção da DQO, mesmo sendo um mesmo sistema de tratamento de efluente.

\section{- Demanda Bioquímica de Oxigênio (DBO)}

A concentração média da Demanda Bioquímica de Oxigênio foi de $306,06 \mathrm{mg}$ de $\mathrm{O}_{2} \cdot \mathrm{L}^{-1}$, o que está dentro da faixa para esgoto bruto $\left(200-750 \mathrm{mg}\right.$ de $\left.\mathrm{O}_{2} \cdot \mathrm{L}^{-1}\right)$, segundo Silva e Mara (1979). No entanto, quando esse esgoto bruto for submetido a um processo de tratamento biológico por lagoas de estabilização em escala piloto do tipo: Lagoa facultativa Lagoa de maturação - Lagoa de maturação, irá ocorrer uma remoção média da DBO da ordem de mais $80 \%$ (SPERLING, 2002). Segundo Vale (2006), a remoção de DBO, em sistema do tipo (LF - LM - LM), foi de $84 \%$ e $76 \%$, Sistema Jardim Lola 1 e Sistema Beira Rio, respectivamente.

Segundo Trajano (1999), a remoção de DBO em lagoas de estabilização é proporcional à carga orgânica superficial aplicada, o tempo que o efluente permanece no reator biológico (TDH), em condições de temperatura e penetração de luz. O que explica diferentes eficiências de DBO para um mesmo sistema. Pode-se concluir que quanto ao lançamento em corpos hídricos, o efluente em questão irá apresentar-se abaixo do limite máximo de concentração $\left(120,0 \mathrm{mg}\right.$ de $\mathrm{O}_{2} \cdot \mathrm{L}$ ${ }^{1}$ ), ou remoção mínima de $60 \%$ (CONAMA 430/11).

\section{Análises Microbiológicas}

\section{- Coliformes Termotolerantes}

A concentração média de coliformes termotolerantes foi de $2,76 \times 10^{6}$ UFC/100mL, o que está dentro da faixa estabelecida para esgoto bruto $\left(10^{6}-10^{9} \mathrm{UFC} / 100 \mathrm{~mL}\right)$, segundo Bastos (2003). No entanto, quando o efluente em questão for submetido a um processo de tratamento biológico por lagoas de estabilização em escala piloto do tipo: Lagoa Facultativa - Lagoa de Maturação - Lagoa de Maturação, irá ocorrer um decaimento da concentração de coliformes da ordem de 99,9\% (SPERLING, 2002). Segundo Vale (2006), a eficiência de remoção de coliformes em um mesmo sistema, foi da ordem de $99,999 \%$.

Na Tabela 2 estão descritas as diretrizes estabelecidas pela WHO (2006) para esgotos tratados na irrigação.

É possível perceber, que quanto maior o número de lagoas de maturação em série, maior será a eficiência do tratamento em relação ao decaimento de grupo de coliformes, cujo principal objetivo dessas últimas lagoas é a remoção de microrganismos patogênicos (SPERLING, 2002).

A eficiência na remoção de coliformes termotolerantes em lagoas de estabilização, está diretamente ligada a fatores como: temperatura; tempo de detenção hidráulica; direção e velocidades dos ventos; incidência de luz; atividade algal.

Pode-se concluir que quanto ao lançamento em corpos hídricos, o efluente em questão irá apresentar-se abaixo do limite máximo de concentração, seguindo as diretrizes propostas da WHO (2006), onde relata que para agricultura restrita, o valor para coliformes é de $1 \times 10^{4} \mathrm{UFC} / 100 \mathrm{~mL}$ e para agricultura irrestrita, o valor deve ser de no máximo $1 \times 10^{3} \mathrm{UFC} / 100 \mathrm{~mL}$. 
Tabela 2 - Diretrizes estabelecidas pela WHO (2006).

\section{DIRETRIZES}

QUALIDADEEFLUENTE

\begin{tabular}{cccc}
\hline $\begin{array}{c}\text { Categoria } \\
\text { Irrigação }\end{array}$ & Opção & $\begin{array}{c}\text { Tratamento de esgotos e } \\
\text { remoção de patógenos }\left(\log _{10}\right)\end{array}$ & E. Coli $100 \mathrm{~mL}^{-1}$ \\
\hline & $\mathrm{A}$ & 4 & $\leq 10^{3}$ \\
Irrestrita & $\mathrm{B}$ & 4 & $\leq 10^{4}$ \\
& $\mathrm{C}$ & 2 & $\leq 10^{5}$ \\
& $\mathrm{D}$ & 4 & $\leq 10^{6}$ \\
$\mathrm{E}$ & 6 ou 7 & $\leq 10^{1}{\mathrm{ou} 10^{0}}^{-10^{4}}$ \\
\hline \multirow{2}{*}{ Restrita } & $\mathrm{F}$ & 4 & $\leq 10^{5}$ \\
& $\mathrm{G}$ & 3 & $\leq 10^{6}$ \\
\hline
\end{tabular}

(A): Cultivo de raízes e tubérculos; (B): Cultivo de folhosas; (C): Irrigação localizada de plantas que se desenvolvem distantes do nível do solo; (D): Irrigação das plantas que se desenvolvem distantes do nível do solo; (E): qualidade de efluentes alcançável com o emprego de técnicas de tratamento tais como tratamento secundário + coagulação + filtração + desinfecção; (F): Agricultura de baixo Nível tecnológico e mão de obra intensa; (G): Agricultura de alto nível tecnológico e, altamente mecanizada; $(\mathrm{H})$ : técnicas de tratamento com reduzida capacidade de remoção de patogênico (por exemplo: Tanque séptico ou reator UASB) associada ao emprego de técnicas de irrigação.

Fonte: WHO (2006).

\section{Caracterização dos Resíduos Líquidos}

Os resíduos líquidos provenientes do bloco $\mathrm{C}$ do Centro de Ciências e Tecnologia da UEPB, após análises físicoquímica e microbiológica, é de esgoto doméstico bruto. Portanto foi proposto um tratamento para tal, a fim de reusá- lo para fins não potáveis, onde merece destaque: Lavagem de piso e rega de jardins. A Tabela 3 apresenta um resumo dos valores médios encontrados nas análises realizadas e os valores de referência do CONAMA 430/11.

Tabela 3 - Média dos parâmetros analisados.

\begin{tabular}{ccc}
\hline Parâmetros Analisados & Valores médios encontrados & $\begin{array}{c}\text { Valores de referência do } \\
\text { CONAMA 430/11 }\end{array}$ \\
\hline $\mathrm{pH}$ & 7,22 & Entre 5 e 9 \\
Alcalinidade & $342,45 \mathrm{mg} \mathrm{CaCO} / \mathrm{L}$ & $120 \mathrm{mg} \mathrm{de} \mathrm{O}_{2} / \mathrm{L}$ \\
\hline DBO & $306,06 \mathrm{mg} \mathrm{de} \mathrm{O} / \mathrm{L}$ & - \\
\hline DQO & $558,57 \mathrm{mg} \mathrm{de} \mathrm{O} / \mathrm{L}$ & - \\
Nitrito & $0,023 \mathrm{mg} / \mathrm{L}$ & $20 \mathrm{mg} / \mathrm{L}$ \\
Nitrogênio Amoniacal & $33,206 \mathrm{mg} / \mathrm{L}$ & - \\
Fósforo Total & $7,26 \mathrm{mg} / \mathrm{L}$ & - \\
Ortofosfato Solúvel & $3,75 \mathrm{mg} / \mathrm{L}$ & $10^{4} \mathrm{ou} 10^{3} \mathrm{UFC} / 100 \mathrm{~mL}$ \\
\hline Coliformes & $2,76 \mathrm{x} 10^{6}$ & $\mathrm{UFC} / 100 \mathrm{~mL}$ \\
\hline
\end{tabular}

\section{Fluxograma do Tratamento Proposto}

A Figura 2 ilustra, esquematicamente, o fluxograma do todo tratamento proposto para o esgoto do bloco C da UEPB, desde o tratamento preliminar, sistemas de lagoas de estabilização (Lagoa Facultativa seguida de duas de
Maturação, em escala piloto), para a reutilização do efluente tratado na própria Universidade (UEPB), pelo fato de ser um processo de tratamento eficaz, de baixo custo de operação, simplicidade e que não requer demanda de energia elétrica.

Figura 2. Fluxograma do tratamento proposto

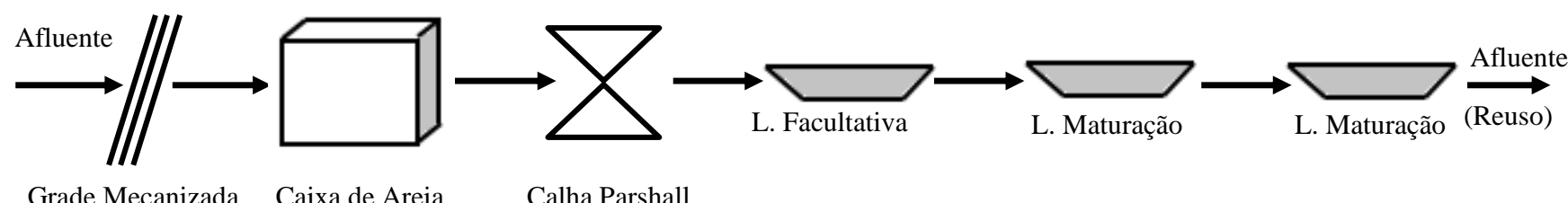




\section{CONCLUSÕES}

Mediante a caracterização do esgoto bruto da UEPB, propõe-se um sistema de tratamento biológico por lagoas de estabilização do tipo: Lagoa Facultativa, seguida de duas lagoas de maturação (em escala piloto), pelo fato de ser o processo de tratamento de esgoto mais simples que se tem conhecimento e não requer demanda de energia elétrica. Portanto, podemos afirmar através de dados coletados que o tratamento proposto, certamente apresentará êxito nos resultados obtidos (remoções). Sendo também influenciada pelas condições climáticas do semiárido, que favorecem consideravelmente o sistema proposto, no que diz respeito, a velocidade de degradação da matéria orgânica carbonácea, como também a remoção destas.

Um esgoto devidamente tratado a ponto de ser devolvido aos rios e aquíferos é suficiente limpo para ser reutilizado em atividades como: Lavagem de pisos, descargas de vasos sanitários, rega de jardins. Com isso, sendo possível desenvolver sistemas de reuso, desde que haja comprometimento dos profissionais encarregados e operado de modo correto dentro de padrões estabelecidos pela legislação vigente, contribuindo de forma direta para o meio ambiente e para as pessoas que dela usufrui.

\section{REFERÊNCIAS BIBLIOGRÁFICAS}

AMBIENTE BRASIL. Reuso de águas urbanas. Disponível em: http://ambientes.ambientebrasil.com.br/agua/artigos _aguas_urbanas/reuso_de_agua. Acesso em 11 de Outubro de 2014.

AQUINO, D. S. Avaliação Eco Toxicológica de Esgotos de Redes Coletoras deum Campus Universitário e da Área Urbana da Cidade de Viçosa - MG e sua Influência na Qualidade dos Corpos Receptores. Dissertação de Mestrado. Programa de Pós-Graduação em Engenharia Civil. Universidade Federal de Viçosa. Viçosa, MG, 46 p., 2013.

ARAÚJO, A. L. C. Comportamento de formas de Fósforo em Lagoas de Estabilização, em escala piloto, sob diferentes configurações, tratando o esgoto doméstico. Campina Grande, 1993. Dissertação de Mestrado - Departamento de Engenharia Civil, Universidade Federal da Paraíba, Campus 2, 1993.

ASSOCIAÇÃO BRASILEIRA DE NORMAS TÉCNICAS. NBR 9648: Estudo de Concepção de Sistemas de Esgoto Sanitário. Rio de Janeiro: Moderna, 5 p., 1986.

ATHAYDE JÚNIOR, G. B.; LEITE, V. D.; ARAÚJO, H. W. C.; SILVA, J. P. B.; SANTOS. V. D.; SOUSA, J. T.; SILVA, W. R. Estudo das Espécies de Fósforo e Nitrogênio em Lagoas de Estabilização. Congresso Interamericano de Engenharia Sanitária e Ambiental, Campina Grande - PB. Artigo. Porto Alegre- RS: ABES, p. $1-8,2000$.

AYERS, R. S.; WESTCOT, D. W. A qualidade da água na agricultura. Tradução de h. R. Ghei. J. F. v Damasceno. 2.ed. Campina Grande: UFPB, 1999. 153p. Estudos

FAO Irrigação e Drenagem, 29 revisado.

AYRES, R. S., WESTCOD, D. Water Quality for Agriculture. Food and Agriculture of the United Nations, Rome, Irrigation and Drainage Paper Number 29. 1985.

BASTOS, R. K. X., MARQUES, M. O. Utilização de esgoto tratado em fertirrigação, hidroponia e piscicultura Uma análise Crítica. Rio de Janeiro - ABES, Rima, 267p. 2003.

BRASIL. Ministério do Meio Ambiente. Conselho Nacional do Meio Ambiente (CONAMA). Resolução n. 430 de 11 de Maio de 2011.

HESPANHOL, I. Potencial de reuso de água no Brasil Agricultura, Indústria, Municípios e Recarga de Aquíferos. Revista Brasileira de Recursos Hídricos. v. 7, n.4, out/dez 2002, p.75-85.

JORDÃO, E. P.; PESSOA, A.C. Tratamento de Esgotos Domésticos, Concepções Clássicas de Tratamento de Esgotos. $2^{\text {a }}$ edição. CETESB, São Paulo, 2005, 544p.

MANUAL DE SANEAMENTO - FUNASA: Engenharia de Saúde Pública - Orientações Técnicas. $3^{\mathrm{a}}$ Ed. Brasília DF: Ministério da Saúde - Fundação Nacional da Saúde, 2004. 407p.

METCALF e EDDY. Wasterwater Engineering - Treatment, Disposal, Reuse. $3^{\text {rd }}$ Edition. McGraw-Hill.1991.

PALMEIRA, G. F. Tratamento Conjugado de Esgoto Doméstico e Lixiviado de Aterro Sanitário em Lagoas de Estabilização. TCC (Graduação) - Curso de Química Industrial, Química, Universidade Estadual da Paraíba, Campina Grande PB, 44 p., 2014.

RIBEIRO, J. Í. C. Proposta de Implantação de um Sistema de Lagoas de Estabilização Conjugadas no Tratamento de Esgoto para Fins de Reuso em Aquicultura e Agricultura na Cidade de Tavares - PB. TCC (Graduação) - Curso de Engenharia Sanitária e Ambiental, Departamento de Engenharia Sanitária e Ambiental, Universidade Estadual da Paraíba, Campina Grande - PB, 69 p., 2014.

SAWYER, C. N.; MC CARTY, P. L.; PARKIN, G. F. Chemistry for Environmental Engineering. $4^{\text {th }}$ edition. McGraw - Hill, 1994.

SILVA, L. K. T. Avaliação preliminar da eficiência de remoção de nutrientes, em três sistemas de lagoas de estabilização no Estado do Rio Grande do Norte. Congresso Norte Nordeste de Pesquisa e Inovação, IFRN, Natal - RN, p. $1-8,2010$.

SILVA, S. A. Manual de Análises Físico-Químicas de Águas de Abastecimento e Residuárias. Campina Grande: Universidade Estadual da Paraíba, 266 p., 2001. 
SILVA, S. A.; MARA, D. D. Tratamento Biológico de Águas Residuárias. Belo Horizonte, UFMG. $2^{\mathrm{a}}$ edição. 196p. 1986.

TRAJANO, A. S. A. Influência das características físicas e operacionais no desempenho de lagoas facultativas secundárias e de maturação. Dissertação de Mestrado, Universidade Federal da Paraíba, 1999.

VALE, M. B. Avaliação da Eficiência da remoção de matéria orgânica e microbiológica de três sistemas de lagoas de estabilização em série na grande Natal - RN: Beira Rio, Jardim Lola 1 e Jardim Lola 2. Monografia (Pós Graduação) - Curso de Engenharia Sanitária, Centro de Tecnologia, Universidade Federal do Rio Grande do Norte, Natal - RN, 94p., 2006.

VON SPERLING, M. Introdução à Qualidade das águas e ao Tratamento de Esgoto: Princípios de Tratamento Biológico de Águas Residuárias. $4^{\mathrm{a}}$ Ed. Belo Horizonte: UFMG, 2002.

VON SPERLING, M. Lagoas de Estabilização: Princípios do Tratamento Biológico de Águas Residuárias. $2^{\mathrm{a}}$ Ed. Belo Horizonte: UFMG, 196 p., 2002.

WHO. Guidelines for the safe use of wasterwater, excreta and greywater. Vol.3. Wasterwater and excreta use in aquaculture. Geneva: World Health Organization, 2006. 